\title{
Habilidades profesionales asociadas a la docencia. Fijando posiciones de profesores en formación
}

\author{
Professional skills associated with teaching, \\ setting positions of pre-service teachers \\ Gerardo Sánchez Sánchez ${ }^{\mathrm{a}}$ Ximena Jara Amigo ${ }^{\mathrm{b}}$ \\ a Doctor en Ciencias de la Educación. Académico adscrito al Departamento de \\ Formación Inicial Escolar de la Facultad de Ciencias de la Educación, Universidad Católica del Maule. \\ Integrante del Centro de Investigación en Educación para la Justicia Social / CIEJUS/ \\ ${ }^{\text {b }}$ Magister en Curriculum y Evaluación. Académica de la Carrera de Pedagogía en Historia, Geografía y Ciencias \\ Sociales. Universidad Autónoma de Chile. Sede Talca \\ $\measuredangle$ gsanchez@ucm.cl,xjaraa@uautonoma.cl
}

\begin{abstract}
RESUMEN
La investigación tiene por novedad aproximarse a la profesionalidad que se construye en el interior de la personalidad del profesor en formación, utilizando la metáfora de la "posición" para hacer visibles y poner en tela de juicio las diferentes perspectivas a través de las cuales significan las habilidades docentes, así como la orientación y postura respecto a la docencia y lo que implica ser profesor.

La metodología asume un carácter cualitativo, basado en una perspectiva interpretativa. Desde la técnica de entrevista abierta, administrada a cincuenta estudiantes de pedagogía primaria. La discusión de los resultados se inscribe en el análisis de contenido.

Los resultados evidencian la construcción de un tipo docente que moviliza habilidades profesionales inscritas en la metáfora del profesor artesano y reflexivo, mediadas fuertemente desde la metáfora del profesor persona.
\end{abstract}

PALABRAS CLAVE: Docencia, formación de docentes, práctica pedagógica, Chile.

\begin{abstract}
Research has the novelty of approaching the professionalism that is built inside the personality of the pre-service teacher, using the metaphor of the "position" to make visible and question the different perspectives through which the teaching skills come
\end{abstract}


to signify, as well as the orientation and view regarding teaching and what it means to be a teacher.

The methodology adopts a qualitative character, based on an interpretative perspective. The technique of open interview, administered to fifty students from an elementary teacher program. The discussion of the results is written in the content analysis.

The results demonstrate the construction of a teaching type that mobilizes professional skills inscribed in the metaphor of the teacher craftsman and the reflective one, strongly mediated from the metaphor of the teacher person.

KEY WORD: Teaching, teacher training, pedagogical practice, Chile

\section{INTRODUCCIÓN}

Toda propuesta formativa se sustenta en un conjunto de reflexiones y supuestos acerca de las tareas que los profesores realizan, razón por la cual diseñar una preparación inicial supone "analizar todas las dimensiones involucradas en el ejercicio del rol, y a partir de ellas definir las cuestiones a ser cubiertas en la formación a fin de posibilitar su desarrollo" (Diker \& Terigi, 2008, p.94).

Asimismo, es necesario reconocer que "la identidad profesional no se forja en el vacío sino en contextos concretos de experiencia y relación: se fragua, en definitiva, en el juego de unas transacciones sociales y biográficas que están sometidas a cambio y evolución" (Altarejos, Ibañez-Martín, Jordán \& Jover, 1998, p. 80).

La profesionalización de una actividad como la docencia exige la consolidación de dimensiones fundamentales.

[....] Por un lado, la dimensión cognoscitiva - todo profesional debe poseer un conjunto de conocimientos y destrezas que le faculten para el ejercicio de su trabajo-; $y$, por otro, la dimensión ético-deontológica - tanto porque uno de los rasgos que caracterizan a las profesiones es la dimensión de servicio, como por la capacidad de autorregulación de la propia actividad que deben asumir sus miembros, con el fin de velar por su recto ejercicio, y defender sus derechos y los de aquellos a quienes van destinadas las actividades profesionales que desempeñan (García \& García, 2012, p.174).

En consecuencia, todo profesor desde el punto de vista cognitivo requiere la adquisición de un conjunto de conocimientos relacionados con el contenido y su didáctica, y en lo referido a su dimensión ética, demanda una responsabilidad considerable pues se trata de un trabajo que incide directamente en la vida de otros seres humanos, poniendo de manifiesto requerimientos de justicia social (Meireu, 2001).

En el encuentro o desencuentro de las dimensiones cognitivas y éticas; profesionales 
y personales, se configura la producción identitaria de los profesores, revistiendo una particular importancia a la hora de definir la naturaleza propia del trabajo docente. Ésta desde la perspectiva histórica ha transitado entre las nociones de vocación, empleo y actitud de vida (García \& García, 2012); desde el punto de vista de los modelos de formación, las opciones se despliegan en un crisol que incluye "el enfoque práctico-artesanal; la tradición normalizadora-disciplinadora; la tradición academicista; el enfoque técnico-eficientista; la concepción personalista, entre otros" (Diker \& Terigi, 2008, p. 110-111); o pensando en paradigmas relativos al oficio de maestro, encontramos

[....] Un maestro ilustrado que domina los conocimientos, un técnico que ha adquirido sistemáticamente los procedimientos técnicos, un practicante artesano que ha adquirido en el terreno ciertos esquemas de acción contextualizados, un practicante reflexivo que se ha construido un saber de la experiencia sistemático y comunicable, más o menos teorizado, un actor social que está comprometido en proyectos colectivos y es consciente de las apuestas antroposociales de las prácticas cotidianas y una persona que está en relación con los demás y en un proceso de desarrollo personal (Paquay \& Wagner, 2010, p. 224).

Estas distintas perspectivas, posiciones o aproximaciones ponen de manifiesto el requerimiento actual de una educación y por tanto formación de profesores, que no puede entenderse como un proceso de "fabricación" del ser humano:

[....] La verdadera revolución copernicana en pedagogía consiste en volver la espalda resueltamente al proyecto del doctor Frankenstein y a la educación como fabricación. La educación, en realidad, ha de centrarse en la relación entre el sujeto y el mundo humano que lo acoge. Su función es permitirle construirse a sí mismo como sujeto en el mundo: heredero de una historia en la que sepa qué está en juego, capaz de comprender el presente y de inventar el futuro (Meireu, 2001, p. 70).

En ese contexto, entendemos la formación de profesores como un proceso de construcción de identidad y al maestro como "profesional de la articulación del proceso de enseñanza -aprendizaje en situación; un profesional de la interacción de las significaciones compartidas" (Paquay, 2010, p. 38).

Coherente con esa opción, "enseñar es utilizar una determinada tecnología y es una práctica concreta, situada siempre en un ambiente de trabajo, que consiste en coordinar diferentes medios para producir unos resultados educativos, en el interior de un determinado contexto" (Tardif, 2004, p. 87). Lo particular de esta tecnología asociada a la enseñanza es que se trataría de una actividad humana, un trabajo interactivo, o sea, un trabajo construido entre personas. Por lo tanto, una visión formativa del trabajo docente para la cual se debe 
preparar al futuro profesor es aquella que nunca deja de lado las condiciones y las limitaciones inherentes a la interacción humana, en especial las condiciones y las limitaciones normativas, afectivas y simbólicas.

Nos parece importante no perder de vista que la enseñanza comparte un mismo espacio con los otros oficios denominados "profesiones del sector humano". En todos ellos debemos enfrentar situaciones sociales complejas, sometidas al tiempo, en las que se combina lo social con lo institucional y lo personal. Ello pone de manifiesto una mejor comprensión de la enseñanza como profesión de lo humano y lo relacional. Por tanto,

[....] Hoy no es posible dejar de señalar, entre las características de la actividad docente, las siguientes: la multiplicidad de tareas que supone el rol docente; la variedad de contextos en que estas tareas pueden desempeñarse; la complejidad del acto pedagógico; su inmediatez; la indeterminación de las situaciones que se suscitan en el curso del proceso de trabajo docente; la implicación personal y el posicionamiento ético que supone la tarea docente (Diker \& Terigi, 2008, p. 96).

Convirtiendo a la enseñanza:

[....] En un oficio que cada vez compromete más a la persona y que expone al individuo en tanto que individuo (y no en tanto que rol profesional). La inversión/ inmersión personal en la tarea (trabajo concreto) puede ser gratificante, pero también puede ocasionar situaciones de depresión, en especial cuando no se cuentan con las competencias y condiciones de trabajo adecuadas y por lo tanto no se alcanzan los resultados esperados (Tenti, 2007, p. 337).

Lo anterior se corresponde con el carácter implicante y envolvente del trabajo docente, entendido como conjunto de interacciones (Tardif, 2004).

En ese sentido, cuando el actor - profesor en formación - se enfrenta a los requerimientos de la práctica y del aula, iniciará necesariamente la búsqueda de una adecuada comprensión del trabajo docente, y de una actuación efectiva capaz de sortear las limitaciones objetivas del contexto donde se desarrolla la tarea, más allá de las prescripciones teóricas o normativas que desde la institución formadora se ha procurado favorecer.

[....] De alguna manera, él tiene que actuar con astucia, tratar de negociar su lugar, hacer frente a las limitaciones y reglas establecidas, investigar estrategias que aumenten sus posibilidades de éxito, etc. Para ello, el actor sólo tiene una "racionalidad limitada", es decir que él posee solamente ciertas informaciones (y no todas) acerca de la realidad social y su inteligencia no puede entonces controlar simultáneamente todos los aspectos de esta misma realidad (Tardif \& Borges, 2013, p. 30). 
Ello implica reconocer que la inserción al aula la realiza desde una percepción situacional reducida y con esquemas de actuación aún incompletos, los cuales "no aparecen de la nada con el inicio de la enseñanza, sino que se van conformando a través de las experiencias prácticas, preformativas y de formación inicial" (Correa, 2008, p. 68); de hecho, se configuran, preferentemente, durante la etapa inicial de inserción a la tarea docente.

En consecuencia, el profesor en formación experimenta la inserción a una profesión (la docencia), en una institución (escuela) y en una cultura (una organización) con lógicas de actuación que exceden su voluntad y poder de acción, enfrentado al desafío personal de otorgar sentido a su experiencia pedagógica.

[....] El rol de las teorías, las prescripciones externas y las experiencias de otros docentes adquiere relevancia cuando ellas pueden articularse con la experiencia del docente y, a la vez, tenga sentido en su trabajo. La formación docente tiene dos funciones: debe enseñar las habilidades reflexivas que todo docente requiere y debe entregarle el discurso y la terminología que permita que los docentes reevalúen y reclasifiquen su experiencia (Fullan \& Hargreaves, 1996, p.178).

En la búsqueda de este sentido, el profesor se encuentra continuamente ocupado en aprender más sobre sí mismo y su manera de trabajar, desarrollando una determinada comprensión de la docencia. Comprensión que surge de la distancia que genera la pretensión de un conocimiento científico y tecnológico orientado a normar la actuación de los profesionales, y los contextos reales de desempeño, donde las prácticas asumen una diversidad insospechada, reproduciendo el esquema de dicotomía conocer y hacer en la práctica, y que bien reportan Schön $(1987,1998,2010)$ como racionalidad técnica, y que Day (2006) denominó concepción aditiva, vale decir, un modelo de aplicación de la teoría.

Con estos elementos teóricos en discusión, se llevó a cabo la investigación cuya novedad es aproximarse a una formación de profesores construida dentro de la profesión, profundizando la comprensión de las experiencias pedagógicas en lo referido a la naturaleza de la profesión, y de las habilidades profesionales asociadas, desarrollada por un grupo de estudiantes que en su proceso de práctica profesional, enfrentan la inserción al trabajo docente fijando una posición respecto a la profesión.

La palabra posición se usa para hacer visibles y poner en tela de juicio las diferentes perspectivas a través de las que los sujetos, en nuestro caso profesores en formación significan las habilidades asociadas a la profesión, utilizando la metáfora de la "posición" para sugerir a la vez la idea de la orientación y de la postura respecto a la docencia y lo que implica ser profesor. Por consiguiente, la posición pretende captar nuestra manera de situarnos y de mirar, y las lentes a través de las que vemos como educadores. Sin olvidar que en los actuales tiempos de perplejidad e incertidumbre, 
[....] La enseñanza es una actividad compleja que tiene lugar dentro de redes de significado social, histórico, cultural y político. A lo largo de la vida profesional, una actitud indagadora proporciona los fundamentos para operar dentro de las culturas cambiantes de la reforma escolar y los planteamientos políticos antagónicos (CochranSmith \& Lytle, 2001, p. 70).

Por esa razón, resulta interesante la comprensión que desarrolla el profesor en formación sobre el conjunto de habilidades docentes que perfilan/configuran/delinean el tipo de profesor al cual aspira, lo cual además condiciona la definición que hagan de la naturaleza de su profesión.

En el escenario actual, los cambios sociales han disparado las demandas a la formación inicial, poniendo de manifiesto la incapacidad de las actuales instituciones de formación para dar respuesta a las necesidades de la profesión docente. Las críticas siguen apuntando a su organización burocratizada, el enfoque esencialmente técnico- instrumental de la formación, el divorcio de la teoría y la práctica, la escasa vinculación con las escuelas. De hecho, "la articulación de la formación disciplinar y pedagógica, y de la formación teórica y práctica, se presentan como debilidades transversales en las resoluciones de acreditación de las carreras analizadas" (CNA, 2018, p. 61). En nuestro país, "la debilidad de la formación inicial docente ha sido refrendada por la Comisión sobre formación inicial docente (Mineduc/OEI, 2005) y el Panel de Expertos (Mineduc, 2010) y como planteáramos antes, por los resultados de los mecanismos disponibles para el aseguramiento de la calidad de la formación inicial (acreditación e inicia)" (Ruffinelli, Cisternas \& Córdoba, 2017, p. 20). Y que en el presente año, a través de la Evaluación Nacional Diagnóstica a los estudiantes de pedagogía (END) se siguen confirmando.

Estas problemáticas son consideradas puntos críticos de la Formación Inicial Docente tanto por investigadores (Ávalos, 2010) como por las diferentes instancias de diálogo técnico y político que se han implementado a nivel gubernamental (Ministerio de Educación, 2005; Consejo Asesor Presidencial para la Calidad de la Educación, 2006; Panel de Expertos para una Educación de Calidad, 2010; Ley 20.903 de 2016, que establece un Sistema de Desarrollo Profesional Docente) con la intención cada vez más compleja de elevar la calidad de la formación inicial docente.

En ese escenario, y desde la perspectiva de Britzman (2003), la presente investigación reconoce en el periodo de la formación inicial docente una oportunidad para centrar los esfuerzos en torno a la reflexión de lo que significa la experiencia práctica en establecimientos escolares como docente.

Por lo tanto, la investigación busca indagar a partir del uso de la metáfora de la "posición" las habilidades profesionales asociadas a la docencia y a lo que implica ser profesor de un grupo de estudiantes de Pedagogía en Educación Básica en proceso de práctica profesional, de la Universidad Católica del Maule. Habilidades que finalmente integran representaciones y teorías personales, esquemas de acción y conductas pedagógicas disponibles. 


\section{METODOLOGÍA}

Diseño: La comunicación que se presenta forma parte de la investigación Los docentes en formación y la comprensión de sus experiencias pedagógicas, desarrollada en el contexto de una Facultad de Ciencias de la Educación, específicamente, de la Universidad Católica del Maule. Un estudio cualitativo, basado en una perspectiva interpretativa (Pérez, 2007), que pretende relevar las nociones de comprensión, diálogo y acción, tratando de resignificar al practicante como actor de su proceso formativo, a partir de la recuperación de algunos insumos del paradigma de la narrativa en las ciencias sociales, en tanto nos interesa analizar las comprensiones que los propios profesores en formación explicitan, en sus relatos orales, de las habilidades docentes y en definitiva del tipo de profesor al que aspiran o creen ser.

El tipo de indagación realizada es transeccional y no longitudinal. Es decir, no seguimos una cohorte en el tiempo con la finalidad de observar sus trayectorias, sino que partimos de un presente determinado (el año 2016) y desde ahí orientamos las entrevistas hacia la recuperación de experiencias presentes y pasadas.

Participantes: El estudio es llevado a cabo en una carrera de Pedagogía que forma profesores de Educación Básica con Mención de una Universidad regional. Considerando criterios de saturación teórica (Strauss \& Corbin, 2002), se desarrollaron 50 entrevistas a estudiantes de educación primaria: 38 mujeres y 12 varones, cuyas edades oscilan entre los 23 y 30 años. Cada uno de los participantes firma su consentimiento para participar voluntariamente del estudio.

Los entrevistados fueron seleccionados considerando, en primer lugar, que se encontraran transitando la etapa final de sus carreras, específicamente su proceso de práctica profesional. Se priorizó, asimismo, el abarcar diferentes perfiles de estudiantes, integrando jóvenes de distintas menciones y en distintos contextos de inserción: urbano y rural. De este modo, para efectos de esta comunicación se pudo acceder a relatos que expresan experiencias de socialización y de formación del grupo en su conjunto.

Instrumento: En este caso fue utilizada la técnica de la entrevista abierta (Flick, 2007) que permite establecer recurrencias y diferencias discursivas en torno a los conflictos a relevar. Se indagó en torno a dos dimensiones de interés: la naturaleza de la profesión docente y las habilidades profesionales requeridas.

Procesamiento de datos: El proceso de análisis estableció tres operaciones importantes, a saber: una fase de reducción de datos en que se selecciona, simplifica y organiza la información para hacerla más abordable, otorgando a cada entrevista un código asociado a la inicial del nombre y un número correlativo; una fase de disposición y reducción de datos, a partir de registro categorial simple, que consideró el levantamiento de cinco categorías de análisis 
(artesano, técnico, reflexivo, personal y social), el que fue acompañado de la evidencia empírica que surgió de las entrevistas, y una fase de obtención de resultados y conclusiones, en que se levantan nuevos elementos y explicaciones relacionadas con los objetivos de la investigación.

\section{RESULTADOS}

Los resultados de la investigación presentan las distintas posiciones identificadas por los profesores en formación en relación a las habilidades profesionales, y desde ellas, la orientación y postura respecto a la docencia y lo que implica ser profesor.

Tabla 1. Posiciones en torno a las habilidades profesionales.

\begin{tabular}{|c|c|c|c|c|}
\hline $\begin{array}{c}\text { Tipo de profesor } \\
\text { (Habilidades profesionales) }\end{array}$ & Opción & Opción & Opción & Total \\
\hline (1) Artesano & $\mathbf{1}$ & $\mathbf{2}$ & $\mathbf{3}$ & 35 \\
\hline (2) Técnico & 29 & 5 & 1 & 9 \\
\hline (3) Reflexivo & 5 & 2 & 2 & 35 \\
\hline (4) Personal & 14 & 16 & 5 & 35 \\
\hline (5) Social & 7 & 17 & 12 & 17 \\
\hline
\end{tabular}

Fuente: Elaboración propia.

Posición práctico / artesanal

Esta posición entiende la enseñanza como una actividad artesanal, donde el conocimiento acerca de la misma supone un proceso de ensayo y error, en el terreno de la acción, y la correspondiente valoración del contacto con la realidad en tanto espacio de aprendizaje de la profesión y del oficio.

Constituye una posición particularmente viva desde la que se orienta la profesión y que en definitiva ejerce notable influencia, permeando el pensar, decir y hacer de los profesores en formación entrevistados.

[...] Este vínculo "no instrumental" que el artesano mantiene con el trabajo y sus resultados le lleva a experimentar un especial compromiso con su entorno laboral: materiales, herramientas y sobre todo con sus aprendices. En la práctica es donde aprendí la mayoría de las cosas, como se dice aprender haciendo y me voy dando cuenta de los errores que presento y los voy mejorando. Soy una maestra autodidacta y buscadora permanente de conocimientos, si bien planifico mis clases igual le doy lugar a la improvisación, porque lo que va surgiendo en clase cambia totalmente lo que hubiera pensado previamente y hace que los estudiantes se motiven y tengan pensamientos propios. Me veo innovando constantemente, siendo creativo y emprendedor 
[...] Pese a creer en una clase estructural y disciplinarmente correcta, ser artesano me permite ser capaz de aceptar que yo también aprendo de mis estudiantes. Como profesor novel creo que es el día a día, el aprendizaje que se genera en el aula, el que te va entregando las herramientas necesarias para hacer que tu clase estructuralmente y disciplinarmente correcta se convierta en una clase centrada en el tipo de estudiantes que tienes en el aula. Es el día a día el que te irá dando las luces de lo que sirve y lo que no tanto, de lo que motiva y de lo que aburre, en definitiva de lo que vale la pena $y$ es realmente importante. Es en la práctica donde me he formado en verdad como una docente...

En la observación del aula y del comportamiento de los estudiantes van descubriendo un sentido distinto de la tarea docente, hasta ese momento insospechado.

[...] Soy ese tipo de persona que observa cada día a sus estudiantes, tratando de responder a las necesidades de todos ellos, al igual que un artesano voy trabajando desde la base de los aprendizajes de los estudiantes, puliendo y lijando sus habilidades y conocimientos de modo de que se forme una persona, voy modelando sus aprendizajes, avanzando paso a paso, corrigiendo los detalles y errores que pudiesen presentarse

[...] Aprendí que no todas las actividades se pueden aplicar a todos los cursos, desechando metodologías brillantes para algunos y pésimas para otros estudiantes, dejando actividades incompletas por volver a mejorar aprendizajes elementales. El aula es un espacio que nos desborda por la complejidad y la inmediatez de las acciones y la diversidad de decisiones. La maestra que declaro ser es fruto de la experiencia práctica, porque si bien la teoría es muy necesaria al momento de enfrentarse a un grupo de estudiantes, la praxis permite desenvolverse con seguridad, actitud, entusiasmo y valentía.

[...] Una de las principales tareas como educadores es estimular el talento del estudiante cuando aún es un diamante en bruto... pues debemos edificar y moldear a cada uno de los niños que se encuentran en nuestras manos, mediante la utilización de la enseñanza más adecuada para que cada uno de nuestros estudiantes sean capaces de confiar en sus habilidades y realizarse como personas. Con los años espero adquirir experiencias en la praxis en aula, mejorando de esta manera su labor.

[...] Mi práctica docente se construye y se adapta en el aula. No es solo la práctica la que se construye en el aula sino el docente mismo. Se puede planificar muy detalladamente una clase y se pueden manejar muchos contenidos pero en la sala de clases siempre se irán dando situaciones que no están planificadas y que no logran prever ya que existe una variable que lo puede modificar todo y son nuestros estudiantes. 
En su inserción al aula, declaran experimentar las complejas tensiones teoría - práctica

[...] Ya que siento que los 5 años de universidad no me han enseñado lo que la práctica me ha entregado en 4 meses, que lo que los profesores nos cuentan en la universidad no se acerca en nada a la realidad, esa realidad cruda que nos golpea la cara a los inexpertos estudiantes que manejamos la teoría, teoría que muchas veces no sirve si no se le agrega el carisma, la empatía, la vocación, el sentido de la realidad.

[...] ¿Quién nos enseña cómo actuar en una sala donde nos encontraremos con diferentes niños y niñas, cada uno con su carga personal; formas de pensar, de sentir, de hablar, de soñar? ¿Quién nos prepara para enfrentarnos ante las difíciles situaciones de violencia, bullyng, acoso, graves faltas de respeto?, ¿Quién nos advierte de lo complejo de la docencia?. El arduo camino de las prácticas pedagógicas, pone de manifiesto que el camino de la pedagogía se recorre de forma solitaria. La práctica profesional llega entonces a enfrentarnos de golpe con la realidad, esa que creíamos conocer. Nos da entonces la oportunidad de hacernos una idea de lo que es ser y sentirse un docente.

La práctica y el ejercicio del trabajo docente emergen como un espacio de aprendizaje e incertidumbre, mediada por el perfil crecientemente diverso del estudiante y de los contextos donde la tarea se lleva a cabo, planteándolo como descubrimiento pero también como foco de tensión.

[...] A través del ejercicio diario de la profesión se van aprendiendo cosas que no te enseñan los libros y las clases teóricas de la universidad. Debemos recordar siempre que trabajamos con personas y cada persona, cada alumno tiene su propio mundo que es complejo y muy distinto al de otro, por ende el profesor prácticamente es un alumno más que debe descubrir cómo funciona ese mundo de cada estudiante, para poder desarrollar un método o una combinación de métodos que ayuden a desarrollar aprendizajes en cada uno de ellos.

[...] Como un artesano que siempre busca innovar en sus creaciones a través de lo que la gente quiere, un profesor también debe buscar nuevas estrategias para enseñar.... $Y$ claro, en muchas de estas iniciativas no he tenido el resultado esperado, ya sea por falta de tiempo, porque no hubo motivación de parte de los estudiantes o porque no fue bien planteada la actividad, en otras ocasiones he logrado satisfactorios resultados. A lo largo del proceso, fui mejorando y desarrollando de mejor manera las actividades de aprendizaje y espero seguir aprendiendo e implementando diferentes maneras de enseñar.

El aula se constituye en el espacio natural de aprendizaje de la docencia, y la comprensión de la profesión, 
[...] Mi trabajo en aula es la base para descubrir mi método de enseñanza, el buscar formas de enseñar, encontrar las preguntas correctas para guiar el aprendizaje de los niños, el trabajo es algo que se debe pulir y cada vez que entro a la sala de clases a trabajar con estudiantes es ahí donde voy aprendiendo la mejor forma de desarrollar mis clases en aula, me gusta relacionar los contenidos escolares con el contexto que se encuentran los alumnos.

Entre más horas en el aula se vayan dando más preparado se puede estar como profesor, es un poco de ensayo y error, cada clase debe ser mejor que la anterior descubriendo las mejores técnicas y generar verdaderos aprendizajes significativos

[...] Aprendo a ser profesor en la práctica, al enfrentar el día a día constato que el trabajo docente es un desafío permanente que tiene relación con nuestra capacidad de sintonizar con las necesidades, siempre diversas de los grupos de estudiantes. Son ellos y sus reacciones los que finalmente movilizan las estrategias de enseñanza, debiendo ser capaz de atender con sensibilidad sus necesidades y requerimientos

En consecuencia, su posición artesanal de la docencia, les permite otorgar una orientación dinámica al desarrollo profesional docente,

[...] La práctica hace al maestro. Un docente siempre está aprendiendo, ya que todos los días se ve enfrentado a situaciones que les hacen pensar y mejorar, experimentando permanentemente en los diferentes contextos para adquirir una mejor experiencia.

[...] En cada práctica ejercida adquirí aprendizajes significativos en relación a lo que es realmente educar. Es en situ donde el docente adquiere los saberes necesarios para desenvolverse como un profesional competente. Se aprende mediante la observación, en la cual nos apropiamos de herramientas, de estrategias, de métodos de enseñanza y de conductas, posibilitando así un desarrollo significativo. Tengo la convicción que la experiencia y las vivencias empíricas cimentan las habilidades del maestro, es decir que mediante los años de práctica como profesora iré adquiriendo aprendizajes que solidificarán mis competencias como docente

Posición técnica

Enfoque que se plantea en términos de una notoria diferencia entre el conocimiento teórico y el conocimiento práctico, con predominio del primero y un abierto cuestionamiento al campo de lo práctico. Este conocimiento experto es universal, no circunscrito a contextos específicos, planteándose como una intervención técnica, de manera objetiva, previsible y eficaz.

En consecuencia, la práctica constituye una actividad aplicada regida por 
prescripciones científicas externamente elaboradas que la condicionan. El docente asume un rol técnico que aprende conocimientos y desarrolla competencias y actitudes adecuadas a su intervención práctica, apoyándose en el conocimiento que elaboran los expertos.

En el discurso de los entrevistados aparece con fuerza la referencia a la planificación, y por tanto a la idea de rigor que debe estar presente en las intervenciones que desarrollan en el espacio del aula.

[...] Planificar implica tomar decisiones previas a la práctica sobre qué es lo que se aprenderá, para qué se hará y cómo se puede lograr de la mejor manera. Si como docentes planificamos según las habilidades y destrezas de nuestros estudiantes mejoraremos el aprendizaje

[...] El sistema y el currículum nos obligan de alguna forma a mantener una estructura sistemática en cuanto a los conocimientos que debemos entregar a nuestros estudiantes, planificando nuestras clases, preparando material adecuado a las necesidades de los educandos

[...] Una docente muy estructurada en todo lo que planifico para una clase, considerando todos los detalles posibles que pudiesen existir al momento de realizar alguna intervención, como por ejemplo el tiempo existente, la cantidad de estudiantes, el contexto en el que se está inserto, el cumplimiento de la estructura de una clase, los recursos a utilizar, logrando ser muy precavida ante todo, con el fin de evitar caer en las improvisaciones y así generar una clase intachable y al pie de la letra de lo señalado en la planificación.

De alguna manera, la importancia otorgada a la planificación pretende reducir márgenes de improvisación, en un trabajo que al comprometer resultados obliga a una gestión eficaz

[...] Centro mi atención en la planificación, me gusta dejar todo establecido, siempre evitando la improvisación. Creo que un docente debe siempre tener su clase preparada, saber el contenido que va a entregar y el material a utilizar y no llegar a la sala a decidir qué hacer.

[...] Considero al momento de hacer mi trabajo, la planificación de este, la metodología que emplearé y que las cosas estén estructuradas. Me gusta un trabajo estructurado, para luego reflexionar y mejorar los errores que cometo o tener presente las cosas que omito. Me gusta el orden y la disciplina al momento de trabajar. Sin una guía, sin patrón me siento un tanto perdida.

[...] Me esmero porque mis clases se acerquen a la perfección, me gustan las cosas estructuradas y que a través de una buena planificación mis estudiantes logren aprender. Detrás de cada clase hay una preocupación previa. 
En esa misma dirección, emerge el imperativo de la competencia asociada al trabajo docente,

[...] Busco ser competente en mi área, planifico con dedicación mis clases, tratando de adelantarme a la realidad de mis alumnos, pensando en cuáles pueden ser las mejores preguntas con el fin de llevarlos a lo que quiero lograr con ellos que es cumplir con los objetivos.

Posición reflexiva

Las referencias a esta perspectiva ponen de manifiesto la complejidad de la enseñanza, pues se desarrolla en contextos singulares, transversalmente matizada por cuestiones éticas y políticas, y por lo tanto de incertidumbre insospechada. Corresponde al docente la capacidad para hacer frente a situaciones únicas, ambiguas, inciertas y por tanto conflictivas en el espacio del aula, adoptando decisiones que resulten adecuadas.

La fluidez que presenta el escenario de la práctica, obliga al docente a combinar la sensibilidad de la experiencia con la investigación teórica, revisando su práctica y buscando nuevas opciones.

El elemento fundamental en la práctica reconocida por los profesores en formación es el desarrollo de la reflexión.

[...] Uno debe reflexionar sobre la manera que enseñamos, debo como profesora asumir muchas responsabilidades, incluso éticas... siendo autocrítica con mi propio trabajo, reconociendo así mis falencias y logrando con ello mejorar mis prácticas y experiencias pedagógicas.

[...] Ya que en mi práctica muchas de las actividades que planifiqué no resultaron de la manera que esperaba, que los niños no reaccionaron como yo pensaba o que simplemente no llamaron su atención y debido a todo ello, tuve que ir cambiando mi metodología de trabajo para las siguientes clases en busca de un mejor resultado.

Solo la reflexión pedagógica lleva a mejorar el trabajo docente ayudándonos a ser mejores profesionales y a mejorar nuestras fallas y fortalecer las buenas ideas.

La reflexión nos ayuda a centrar nuestras clases y hacerlas más cercanas a nuestros estudiantes según sus características personales convirtiendo una clase común $y$ corriente en una significativamente importante para los estudiantes

[...] El profesor debe ser un sujeto flexible en la labor que desempeña, ya que debe ser capaz de lidiar con los diversos problemas y acontecimientos que ocurren dentro del ejercicio docente para crear un ambiente de trabajo agradable y motivador para los jóvenes que se están formando.

Un ente que continuamente evalúa su desempeño y modifica aquellos aspectos que 
no favorecen el proceso de enseñanza - aprendizaje de sus estudiantes, todo esto con la intención de ir mejorando la práctica. Ya que si un niño no aprende de la manera que les enseñamos, debemos enseñarle de la manera que ellos aprendan.

[...] Es importante en todo el trabajo docente reflexionar sobre la práctica para mejorarla. Las características de los estudiantes y la complejidad de los contextos obligan a estar permanentemente revisando nuestras acciones. Eso no resulta una tarea fácil, sin embargo, genera una reinvención continua.

Este imperativo de la reflexión lo asocian a la naturaleza compleja de la práctica y la docencia que deben desarrollar.

[...] Son muchas las situaciones a las que nos enfrentamos cuando nos convertimos en educadores, y definitivamente la labor docente no es tarea para cualquiera. No se trata simplemente de ponerse de pie al frente de unos cuantos niños, apoyándonos de materiales y recitando casi de memoria algunos párrafos. Ser profesor es hacer frente a muchos retos derivados de la interacción con otros. Una profesora capaz de reconocer sus puntos fuertes y sus debilidades. Que intenta corregir y mejorar aquello que no le funciona, que revisa de manera continua su quehacer, que tiene inquietudes y que se enriquece con lo que le entregan sus estudiantes y colegas que tienen más experiencia. Que entiende que en la pedagogía nunca se termina de aprender y que siempre se está en constante formación.

[...] Durante mi práctica tuve que ir probando metodologías con los distintos cursos con los que trabajé y tuve que adaptarme a las distintas maneras de trabajar que tenía cada curso. Tuve que experimentar y reflexionar en conjunto respecto a los métodos de enseñanza, tuve que equivocarme y pase por momentos frustrantes pero el ser practicante reflexivo me hace ser flexible y me da capacidad de adaptarme a los distintos problemas que puedan ir surgiendo.

Esta reflexión referida a un trabajo de naturaleza compleja, puede tener distintos focos de preocupación, entre los cuales figuran la persona del docente o el alumnado y su aprendizaje.

[...] Constantemente me cuestiono sobre mi papel como docente, en el cómo lo estoy haciendo y qué podría mejorar, además de recibir siempre aquellas críticas constructivas de una manera positiva. También intercambiando ideas con mis pares sobre qué es lo más factible para el aprendizaje significativo de mis estudiantes, pues sé que siempre estaré absorbiendo nuevos conocimientos, estrategias y metodologías que me ayudarán a mejorar mi proceso como docente.

[...] Evaluar mis errores, aciertos, ventajas y desventajas. Necesito ser capaz de revisar lo que estoy haciendo en términos del impacto que mis decisiones tienen en el aprendizaje de los estudiantes. 
[...] Un profesor debe contar con la capacidad y las herramientas para realizar una reflexión de su práctica docente, de forma efectiva y pertinente, pensando en los intereses, habilidades y motivaciones de sus estudiantes, además de evaluar su contexto, informarse en relación a las habilidades sociales del sujeto, interesarse por la forma en que piensan sus estudiantes

Finalmente, advierten la potencialidad que representa la reflexión en la docencia y en su propio desarrollo profesional, favoreciendo la capacidad de toma de decisiones y otorgando la posibilidad de mejorar.

[...] Incluyo tanto los aprendizajes teóricos adquiridos en mi proceso de formación como las enseñanzas surgidas en la práctica. Reflexiono cómo influyen e impactan en los alumnos tanto las metodologías usadas, como los recursos empleados, seleccionando las más adecuadas según las características propias de cada estudiante... comprender el accionar docente y tomar decisiones en los momentos que se requiera, flexibilizando la enseñanza.

[...] Ejercicio mal ponderado pero que trae progresos en las actuaciones docentes, pues incentiva que el profesor al verse ante diversas situaciones en el aula, pueda moldear su comportamiento y actuación en función de las utilidades de aprendizaje.

El profesor se transforma desde un aplicador fundamentalista de las metodologías aprendidas en sus cátedras universitarias, a un creador de nuevas metodologías de aprendizaje y trabajo en el aula. En una profesión que trabaja con personas y con la diversidad debemos estar permanentemente investigando los aciertos y también los errores, para lograr avanzar.

[...] Aprendo de mis errores y también de lo que me entregan mis estudiantes. Cada vez que alguna actividad no resulta como lo esperaba, comienzo a analizar los errores o falencias que se pudieron hacer presentado, también comienzo a pensar cómo puedo mejorar las actividades según las habilidades o destrezas de mis estudiantes. Analizar me ayuda a identificar como ha sido el trabajo que he realizado, saber si ha sido efectivo o no, de esta manera puedo mejorar constantemente.

\section{Posición Personal}

Esta posición sitúa el sentido de la tarea docente en las habilidades personales del profesor como garantía de la futura eficacia docente, y estableciendo distancia respecto a los enfoques de corte técnico y su foco en el desarrollo de habilidades o competencias.

Desde la perspectiva de los profesores en formación, el recurso más importante de la docencia es el profesor mismo, y por lo tanto el proceso de avance que cada uno logra en relación a la tarea a desarrollar. 
[...] Soy una profesora en crecimiento que espera que con el error pueda aprender algo nuevo, para así mejorar mi calidad como docente. Siempre estoy en crecimiento, evoluciono y me transformo y puedo pulir mis habilidades desarrollando otras. Lo anterior repercute directamente en la relación que se entabla con los alumnos, pues el auto conocerse permite aprender del otro, a valorar la diversidad de pensamientos, habilidades... incluir en el desarrollo de las clases la heterogeneidad presente en el salón.

[...] Nuestras actitudes y habilidades personales influyen en el tipo de profesor que somos, ya que en situaciones conflictivas e imprevistas se suele reaccionar en base a nuestros pensamientos incluso ante que la teoría. Entendí que al momento de hacer una clase no hay que obligarse a ser una persona que no eres si no potenciar incluso aquellas cosas negativas en tu personalidad puede contribuir a realizar el trabajo docente.

[...] Mucho antes de ser docente soy una persona, un ser como cualquier otro, con virtudes y defectos. Ser docente va más allá de la transmisión de conocimientos teóricos o solo conceptos a nuestros estudiantes, debemos formar personas, impactar en las vidas de nuestros estudiantes. El profesor sea un ejemplo positivo para sus estudiantes, un ejemplo positivo en su formación

En ese sentido, el acento de la formación y el trabajo docente se sitúa en un conjunto de disposiciones que movilizan la afectividad, las actitudes y el cambio personal.

[...] Creo que los valores y las buenas actitudes generan en los estudiantes sentimientos de seguridad, el sentirse querido, aceptado, respetado y comprometido.

Al trabajar con seres humanos debe encontrarse en primera instancia la característica de "humano" que toda persona posee.

Un profesor es un ejemplo a seguir para sus estudiantes, los niños imitan las actitudes que ven en los adultos... siendo el profesor un modelo en sus vidas, por lo tanto su actitud frente a la clase debe ser acorde a la situación en la que se encuentra y al público con que se presenta.

[...] Este conjunto de características: respetuoso, empático y solidario es el que debiera poseer todo profesional, pues no podemos dejar de lado que antes de cualquier título somos seres humanos, por lo cual nos debemos respetar entre sí, conocernos y aceptarnos. Es de esta manera, que como docente tendré la capacidad de conocer a mis estudiantes, respetando sus fortalezas, dificultades, sueños, miedos.

[...] Capacidad de ser empáticos, de amar, ayudar y creo que esa es la base de la verdadera acción educativa. Se trata de formar íntegramente y eso me involucra necesariamente en los que soy como persona y lo que logro proyectar. El profesor no debe olvidar que trabaja con personas que están en proceso de crecimiento. 
Esta forma de comprender el sentido de la formación se sustenta en el reconocimiento y valoración de los estudiantes como sujeto de su propio proceso educativo.

[...] No debemos olvidar que trabajamos y enseñamos a seres humanos, personas que piensan, sienten y opinan de manera diferente y hay que estar siempre atento a como ellos van aprendiendo y ser un apoyo en su formación.

[...] Cada profesor tiene que saber y conocer a todos los estudiantes que tiene en el aula, que tiene que comprender por lo que está pasando, sabiendo que no es fácil debido a que son muchos los estudiantes y cada uno de ellos tiene necesidades distintas. Detrás de cada uno de ellos hay una historia, que explica el aprendizaje. Lo viví en mi práctica profesional donde vi a alumnos que no trabajan debido a que los padres no están presentes para reforzar conocimientos, o que trabajaban todo el día y el alumno solo pasaba solo, además pude notar la diferencia de alumnos que están psicológicamente bien con su entorno y sus familiares, a alumnos que no estaban bien debido a que se les había fallecido su madre o padre, imaginándose el dolor para un alumno o para cualquier ser humano... Quiero ser ese tipo de profesor consciente de lo que le pasa a cada alumno, una profesora dispuesta a entregar contenidos y apoyo a sus estudiantes. Ejemplo de profesor persona...

En esa perspectiva, se releva el papel de modelaje que compromete el trabajo docente.

[...] Se dice que el maestro es el modelo a seguir de los estudiantes, por lo que yo no pretendo mostrar como ejemplo a un docente cuadrado, frustrado, obsesionado con la perfección...

[...] Quiero dejar una huella imborrable en la vida de cada sujeto, quiero ser un modelo a seguir y trascender de esa manera en la vida de cada sujeto,... lo primordial es saber escuchar y hacer sentir a cada uno de los estudiantes que su opinión vale la pena, siento que actúo como un agente motivador y facilitador, enfocándome en la transversalidad, generando altas expectativas en sus convicciones acerca de su futuro, con el fin de que logren desarrollarse como personas íntegras.

[...] A medida que avanzaron las prácticas, con ello la experiencia y la visualización de diferentes contextos sociales, conocí la vulnerabilidad de los niños, sin importar el tipo de colegio, siempre estaba ahí, en estudiantes descendidos en sus calificaciones, a la vez muy inteligentes. Ser para ellos un modelo a seguir. Procuran que descubran el sentido de aprender lo que les estoy ensañando.

Posición Social

Esta perspectiva releva el rol social del maestro imbuido en proyectos colectivos 
y en los debates sobre la profesión y la educación. Se trataría de un profesor capaz de analizar el sistema en sus múltiples dimensiones y comprometido en la idea de transformación social; más consciente de las problemáticas que aquejan a la escuela y al sistema educativo.

En el discurso de los profesores en formación, emerge con fuerza la idea de transformación social desde la tarea desarrollada por los docentes.

[...] Aquel profesor que espera enseñar más que matemáticas, una forma de vivir, una forma de enfrentar la vida, una forma de actuar ante quienes nos rodean, a vivir la vida de la forma más digna posible, con valores y un sentido de comunidad que rompa fronteras establecidas. Se trata de cambiar el mundo.

[...] Un ser social en pro de una educación con equidad y calidad. Una profesora innovadora y abierta al cambio educativo, que busca el cambio y la transformación social.

[...] Tratar de cambiar el mundo a partir de la educación de los más pequeños. Sentía que la sociedad es tan individualista que ni siquiera se preocupaban de las personas que necesitan ayuda. Trato de concientizar a mis alumnos, desarrollando un sentido ecológico.

[...] Considero que esta debiera ser una labor innata dentro de nuestro trabajo, entender que somos sujetos de cambio, que si podemos lograr transformaciones significativas en un niño y que por ende, nos debemos hacer cargo de estos cambios. Contribuir con nuestra acción a formar sujetos que transformen su entorno y la sociedad.

Complementariamente también se plantea la idea de compromiso con el sujeto para su inserción social.

[...] Quiero formar personas con las capacidades y habilidad que les permitan desenvolverse y afrontar de buena manera los diversos obstáculos en sus vidas, que sean resilientes frente a la adversidad y los cambios.

[...] Nuestra misión no es formar máquinas, sino formar personas que posean las competencias, para desempeñarse en la vida de forma efectiva, hoy nuestra sociedad necesita más personas y menos robots, está en manos de nosotros como profesores transformar esta sociedad actual.

[...] Dado los contextos con un alto porcentaje de estudiantes vulnerables

No es posible estar en el campo educacional y social de una forma neutra, mi condición mi trabajo mi responsabilidad me obligan necesariamente a tomar decisiones que aseguren las habilidades que los alumnos necesitarán para enfrentar la realidad social.

[...] Me propuse nunca olvidar que en los quehaceres propios de un oficio, 
interactuaba con personas. En las manos del profesor está la posibilidad de mejorar realidades, de construir nuevos caminos y de avanzar hacia los sueños, que debe ser ejercida por personas comprometidas con la labor educativa.

\section{DISCUSIÓN Y CONCLUSIONES}

La docencia al igual que todas las profesiones se configura por la delimitación de un ámbito propio de actuación; y la necesidad de una preparación específica para su ejercicio expresado en habilidades, estándares de desempeño o competencias según sea el enfoque adoptado y las demandas sociales.

En la especificidad de la docencia emerge la referencia a dos dimensiones que resultan fundamentales. Desde una parte, la dimensión cognitiva que asegura en el profesor el conjunto de conocimientos y las destrezas requeridas para el ejercicio de su labor; y desde otra, la dimensión deontológica, asociada a la responsabilidad que implica el trabajo con personas, ya que la acción desarrollada incide profundamente en la vida de otros seres humanos.

La evidencia muestra en general una preocupación decidida y prescriptiva en la primera de las dimensiones y menos resuelta en relación a la segunda, con las implicancias que cuando el profesor en formación se enfrenta al ejercicio de la profesión lo hace desde ciertos proyectores de naturaleza conceptual con los cuales busca capturar insatisfactoriamente la especificidad de una tarea que se le escapa y que lo obliga a desarrollar nuevas y más situadas compresiones, teniendo con ello la posibilidad de poner en tela de juicio las posiciones hasta el momento desarrolladas. En este último escenario emergen las llamadas habilidades profesionales cuya posición marca determinadas aspiraciones y concepciones respecto a la naturaleza de la profesión y su ejercicio. Desde la perspectiva de Charlier (2010), las habilidades profesionales "son el resultado de la articulación de tres registros de variables: los conocimientos, los esquemas de acción, y un repertorio de conductas y rutinas disponibles (p. 147). Vale decir, incluirían los conocimientos, las representaciones, las teorías personales y los esquemas de acción movilizados para resolver los problemas dentro de la situación de trabajo, y por lo tanto, desde ellas se configuran determinadas posiciones respecto a la docencia.

Enfrentado a los desafíos de la práctica, este grupo de profesores no escapa a este imperativo de la definición de la naturaleza y especificidad de la docencia y bien lo reflejan sus posiciones, las cuales aparecen fuertemente mediadas desde la práctica, específicamente por la experiencia que implica la inserción en los contextos escolares.

La posición que el profesor en formación desarrolla del conjunto de habilidades docentes desde las cuales tienden a configurar el tipo de profesor al que aspira, ponen de manifiesto una comprensión de la docencia en las expresiones de "complejidad", "interacción" y "contextual", las cuales son correspondientes con el planteamiento de Altet (2010) para el que el profesor es "un profesional de la articulación del proceso de enseñanza - aprendizaje 
en situación; un profesional de la interacción de las significaciones compartidas” (p. 38), y de Tardif, (2004) para quien la docencia refiere a "una actividad que se manifiesta en concreto en el ámbito de las interacciones humanas y lleva consigo, inevitablemente, la señal de las relaciones humanas que la constituyen" (p.86). Para este grupo de profesores en formación, la tarea docente se desarrolla en un medio ecológico que resulta complejo y en un escenario comunicativo vivo y cambiante que involucra la interacción de factores y condiciones variadas. Así, en el ecosistema escolar se enfrentan a problemas que en gran medida se resuelven enfrentados a dilemas de la práctica, de mucha indeterminación y complejidad, que no pueden ser asumidos desde la prescripción o la receta preestablecida.

En la definición de posiciones que realiza este grupo de profesores en formación, se plantea la noción de docencia como actividad que moviliza preferentemente la referencia a conceptos de vocación y actitud vital, más que a expresiones de empleo. Representan la docencia como respuesta a una vocación que supone una preocupación por los demás y los efectos perfectivos sobre los sujetos que se atienden y la conciencia de esforzarse personalmente por ser mejor (García \& García, 2012). Asimismo, evidencian la estrecha relación entre los ámbitos profesionales y personales para el desarrollo de la docencia, exigiéndoles una actitud que esencialmente los compromete como persona.

En ese contexto, los profesionales en formación, construyen posiciones en torno a la docencia, y por tanto de las habilidades profesionales que involucra, en torno a la metáfora “artesanal" y "reflexiva”, mediadas fuertemente por la idea de una profesionalidad que se construye en el interior de la "personalidad" del profesor en formación. Asumiendo un carácter bien transversal en la definición de posición en torno a la docencia, la metáfora "personal" demanda del profesor implicación en la tarea. Aquí radica la fuente de satisfacción, pero también de peligros que encierra la docencia, pues supone entrar en una relación afectiva en la que no podemos no estar implicados (Meireu, 2001). Para este grupo de profesores, esta constituye una dimensión de la docencia que se descubre y confirma esencialmente en la práctica, sobre el terreno.

La metáfora artesanal, pone de manifiesto el saber práctico entendido ante todo como un esquema de acción necesario que se adquiere sobre el terreno, en nuestro caso la escuela y el aula. La posición reflexiva, enfatiza un saber de la experiencia teorizado que permite analizar situaciones, analizarse en situación, evaluar estrategias y crear dispositivos de acción. Finalmente, desde la metáfora personal, la docencia requiere tomar conciencia de su estilo personal, iniciando así una dinámica de desarrollo personal.

Dada la complejidad que representa la docencia, no es posible un reduccionismo tal que lleve a la presencia de posiciones únicas o unidimensionales, muy por el contrario, se visualiza la presencia de mixturas y matices dados por la referencia recurrente al par artesanal - reflexivo; artesanal- persona; y el personal- reflexivo. Se trataría de una forma de entender la docencia cuyas áncoras están ancladas a la práctica y su multidimensionalidad. Ello constituye un aprendizaje a tener presente en los procesos de formación inicial, donde 
la preparación para la docencia debería ser abordada en relación directa a su complejidad y tratando de "valorar las actitudes y aptitudes que dan cuenta de la globalidad intrínseca del oficio de enseñar, más que de sumar minuciosamente habilidades múltiples" (Bélair, 2010, p. 106).

Los resultados de la investigación confirman el espacio de la práctica y la inserción a los contextos escolares como el ámbito propicio para el aprendizaje de la profesión y su correspondiente desarrollo. Desarrollo que en general conciben como evolutivo y dinámico, demandando el compromiso de la actualización permanente. Para la formación inicial desarrollada desde la institución universitaria, ello supone valorar un acercamiento al contexto escolar empleando de manera simultánea, la acción y la reflexión. Y para el profesional en formación, la posibilidad cierta de valorar la toma de conciencia acerca de sus propios esquemas de acción y del carácter singular de éstos como base para la comprensión de la tarea docente.

Para la formación inicial, el estudio aporta novedad tendiente a generar un dispositivo de trabajo orientado a personalizar y contextualizar la formación, proporcionando diversidad de experiencias desde las cuales sea posible el poner en tela de juicio los esquemas de pensamiento y acción que comienzan a elaborar los futuros profesores, y que se movilizan para bien o para mal enfrentados a los desafíos del aula y de la práctica con estudiantes y contextos reales. El desafío que enfrenta la formación inicial de profesores es contribuir a disminuir las brechas entre el trabajo prescrito o normativo y las zonas de indeterminación que caracterizan el trabajo docente real, y para ello resulta crucial detenerse en los posicionamientos que sobre la docencia comienzan a adoptar los futuros profesores. Conscientes de la necesidad de "familiarizar al aprendiz con unos juegos de reglas flexibles y cambiantes, en protegerlo frente a la ilusión de la explicación teórica todopoderosa, falaz e indispensable por el hecho de ser parcial y estereotipada" (Carbonneau y Hétu, 2010, p. 123). De ahí la importancia de impulsar, en la etapa de formación, condiciones para el desarrollo de las habilidades profesionales a través de la práctica, pues ésta constituye el punto de partida y el puntal de su reflexión, ya que el sujeto, directa o indirectamente frente a la realidad que enfrenta, se posiciona como actor con determinados niveles de autonomía profesional.

\section{REFERENCIAS}

Altarejos, F., Ibañez-Martín, J. A., Jordán, J. A., \& Jover, G. (1998). Ética docente. Barcelona: Ariel.

Altet, M. (2010). La competencia del maestro profesional o la importancia de saber analizar las prácticas. En L. Paquay, P. Perrenoud, M. Altet, \& E. Charlier (Eds.), La formación profesional del maestro. Estrategias y competencias (pp. 33-54). México: FCE.

Ávalos, B. (2010). Formación inicial docente en Chile: calidad y políticas. En C. Bellei, D. Contreras, \& J. Valenzuela (Eds.), Ecos de la revolución pingüina. Avances, debates y 
silencios en la reforma educacional (págs. 257-284). Santiago de Chile: Universidad de Chile/UNICEF.

Britzman, D. (2003). Practice Makes Practice: a Critical Study of Learning to Teach, Revised Edition. NY: State University of New York Press.

Bélair, L. (2010). Enseñar la complejidad del oficio de maestro. En L. Paquay, p. Perrenoud, M. Altet \& E. Charlier (2010). La formación profesional del maestro. Estrategias y competencias (pp. 88-106). México: FCE.

Cochran-Smith, M., \& Lytle, S. (2001). Más allá de la certidumbre: adoptar una actitud indagadora sobre la práctica. En A. Lieberman \& L. Miller (Eds.), La indagación como base de la formación del profesorado y la mejora de la educación (pp. 65-79). Barcelona: Octaedro.

Carbonneau, M., \& Hétu, C. (2010). La formación práctica de los maestros y el nacimiento de una inteligencia profesional. En L. Paquay, P. Perrenoud, M. Altet \& E. Charlier (Eds.), La formación profesional del maestro. Estrategias y competencias (pp. 107-138). México: FCE.

Charlier. E. (2010). Cómo formar maestros profesionales. Por una formación continua vinculada con la práctica. En L. Paquay, P. Perrenoud, M. Altet \& E. Charlier (Eds.), La formación profesional del maestro. Estrategias y competencias (pp. 139-169). México: FCE.

Comisión Nacional de Acreditación (2018). Carreras de pedagogía: análisis de fortalezas y debilidades en el escenario actual. Serie Estudios sobre Acreditación. Recuperado de: http://www.investigacion.cnachile.cl/

Consejo Asesor Presidencial para la Calidad de la Educación. (2006). Informe Final. Santiago de Chile: Consejo Asesor. Ms.

Day, C. (2006). Pasión por enseñar. La identidad personal y profesional del docente y sus valores. Madrid: Narcea.

Diker, G., \& Terigi, F. (2008). La formación de maestros y profesores: hoja de ruta. Buenos Aires: Paidós.

Correa, E., Colette, M., \& Rittershaussen, S. (2008). Vers une conceptualisation de la situation de stage: explorations internationales. Montreal: Editions du CRP.

Flick, U. (2007). Introducción a la investigación cualitativa. Madrid: Morata.

Fullan, M., \& Hargreaves, F. (1996). La escuela que queremos. Buenos Aires: Amorrortu Editores.

García, M., \& García, J. (2012). Filosofía de la educación. Cuestiones de hoy y de siempre. Madrid: UNED.

Meireu, PH. (2001). Franskenstein educador. Barcelona: Laertes.

Ministerio de Educación, Mineduc (2005). Informe Comisión sobre Formación Inicial Docente. Santiago de Chile: Serie Bicentenario.

Ministerio de Educación, Mineduc (2006). Ley N² 20.129. Establece un Sistema Nacional de 
Aseguramiento de la Calidad de la Educación Superior. Santiago: Gobierno de Chile. Ministerio de Educación, Mineduc (2016). Ley N ${ }^{\circ}$ 20.903. Crea el Sistema de Desarrollo Profesional Docente y Modifica otras Normas. Santiago: Gobierno de Chile.

Panel de Expertos para una Educación de Calidad. (2010). Informe Final: Primera Etapa. Propuestas para fortalecer la profesión docente en el sistema escolar chileno. Santiago de Chile: Ministerio de Educación.

Paquay, L., \& Wagner, M. (2010). Formación continua y videoformación: que habilidades se deben priorizar. En L. Paquay, P. Perrenoud, M. Altet \& E. Charlier (Eds.), La formación profesional del maestro. Estrategias y competencias (pp. 222-263). México: FCE.

Paquay, L., Perrenoud, P., Altet, M., \& Charlier, E. (2010). La formación profesional del maestro. Estrategias y competencias. México: FCE.

Pérez, G. (2007). Investigación cualitativa. Madrid: La Muralla.

Ruffinelli, A., Cisternas, T., \& Córdoba, C. (2017). Iniciarse en la docencia. Santiago de Chile: Ediciones Universidad Alberto Hurtado.

Schön, D. (1987). La formación de profesionales reflexivos. Barcelona: Paidós - MEC.

Schön, D. (1998). El profesional reflexivo. Cómo piensan los profesores cuando actúan. Barcelona: Paidós.

Schön, D. (2010). La formación de profesionales reflexivos: Hacia un nuevo diseño de la enseñanza y el aprendizaje en las profesiones. México: Paidós.

Strauss, A., \& Corbin, J. (2002). Bases de la investigación cualitativa. Técnicas y procedimientos para desarrollar la teoría fundamentada. Bogotá: CONTUS - Editorial Universidad de Antioquía.

Tardif, M., \& Borges, C. (2013) Lainserción profesional de docentes noveles: tendencias recientes y retos futuros. En E. Correa, M. Cividini, R. Fuentealba \& I. Boerr (Eds.), Formación e inserción profesional: desafíos y pistas de facilitación para la profesionalización docente (pp. 19-43). Santiago: SDL Impresores.

Tardif, M. (2004). Los saberes docente y su desarrollo profesional. Madrid: Narcea.

Tenti, E. (2007). Consideraciones sociológicas sobre profesionalización docente. Educ. Soc., Campinas, 28(99), 335-353. 
